\title{
The enhanced high speed inboard pellet fuelling system at ASDEX Upgrade
}

\author{
B. Plöckl ${ }^{1}$, P. T. Lang ${ }^{1}$, G. Sellmair ${ }^{1}$, J. K. Stober ${ }^{1}$, W. Treutterer ${ }^{1}$, I. Vinyar ${ }^{2}$, ASDEX Upgrade team ${ }^{1}$ \\ ${ }^{1}$ Max Planck Institute for Plasma Physics, EURATOM Association, Boltzmannstr. 2, 85748 Garching, Germany \\ ${ }^{2}$ PELIN LLC, 27A, Gzhatskaya Ulitsa, St. Petersburg, 195220, Russia
}

\begin{abstract}
After more than 20 years of operation and numerous modifications, a substantial modernisation of the inboard pellet injection system on ASDEX Upgrade was carried out. This enhancement was necessary to meet new functional requirements and to benefit from technical progress as well. New requirements are the integration in Discharge Control System, variation of settings of pellet train parameters during one discharge, temperature control of ice extrusion and overcome process limits caused by out-dated vacuum components. The Programmable Logic Controller system is now fully migrated to S7-300 using High Speed Boolean Processor FM352-5 to replace hardwired logic elements and enable flexible control of process parameters. First applications on ASDEX Upgrade concerning high density operation and ELM control are presented to confirm usefulness of implemented features.
\end{abstract}

Keywords: Tokamak, ELM Mitigation, Pellet pacing, Pellet fuelling, Centrifuge launcher

\section{Introduction}

The pellet injection system is composed of a centrifuge based accelerator with a "looping" guiding tube system [1]. There are two cryostat systems, one for extrusion of a rectangular ice rod with a cross section of $1,4 \mathrm{~mm}^{2}, 1,65 \mathrm{~mm}^{2}$ or $1,9 \mathrm{~mm}^{2}$. This ice is stored in the second cryostat at a temperature of about $5-10 \mathrm{~K}$. The pellets are produced on demand during the pellet train. The ice rod is pushed forward by an arm, operated by a stepping motor. The number of steps $(0,25 \mathrm{~mm} / \mathrm{step})$ defines the length of the pellet. Standard lengths connected to the pellet size are: $1.5 \mathrm{~mm}, 1.75 \mathrm{~mm}$ and $2.0 \mathrm{~mm}$. The pellet is cut from the pushed ice rod and falls down in a stop cylinder which defines the start of acceleration by the acceleration arm.

At the end of the acceleration arm the pellet runs through a funnel in the transfer line, which guides the pellet to the high field side (HFS) of ASDEX Upgrade (AUG). The injection angle is $72^{\circ}$ w.r.t. horizontal plane. The overall path length is $17 \mathrm{~m}$; pellet speed is from $240 \mathrm{~m} / \mathrm{s}$ to $1000 \mathrm{~m} / \mathrm{s}$. The three standard sizes correspond to an amount of $1.6 \times 10^{20} ; 2.7 \times 10^{20}, 4.1 \times 10^{20} \mathrm{D}$ atoms. The mass loss of pellet during transit through guiding tube is up to $50 \%$, depending on pellet speed and size.

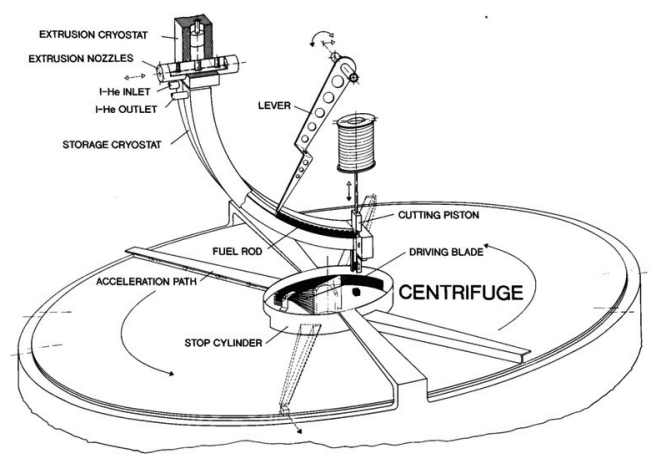

Fig. 1: Extrusion cryostat, storage cryostat, movable 3-way nozzle, lever and cutter.

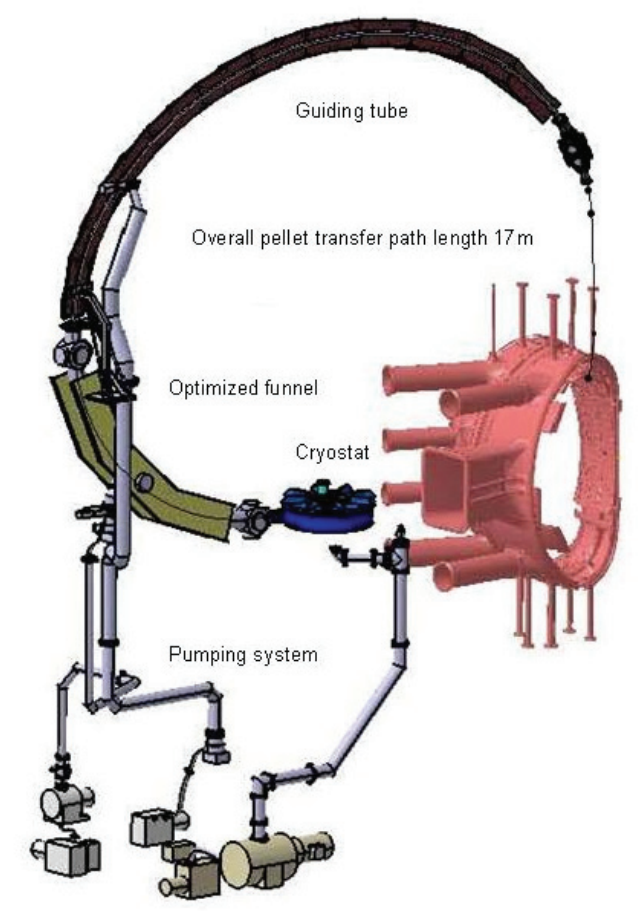

Fig.2: High Speed inboard pellet injection system at ASDEX Upgrade composed of cryostat, centrifuge, transfer looping and vacuum system in AUG Seg.5.

\section{Modifications and enhancements}

The existing system, limited by technical constraints and wear and tear after partly more than 20 years of operation, was subject to a substantial modernisation, vacuum components and control system were renewed.

The turbo molecular vacuum pumps (classic design) of the transfer system were replaced by compound pumps (Pfeiffer HiPace700) with a much higher compression rate especially for light gases. All vacuum gauges were replaced by digital Pfeiffer transmitters equipped with PROFIBUS interface. Most valves of the 
gas supply and vent system are replaced to compensate wear and tear as well.

The control system has been deeply modernized. The logic of the existing system was partly hard wired with relays, timing and logic units. Only a few very particular control units are kept and reintegrated in the new design. The control system is now consistently galvanically separated from the torus hall potential. All components are connected via PROFIBUS; the communication to the control room is served by an optical link.

The Programmable Logic Controller (PLC) is SIMATIC S7-300, located in the control room. Due to the dimensions of the system, peripheral devices are installed: ET200M and ET200S with valve unit Bürkert 8644 AirLINE.

\section{New features of PLC concept}

The feed of the ice rod is driven by a stepping motor, which is controlled in single step mode. The step frequency is $1 \mathrm{kHz}$. The stepping signal just as the cutting request is generated by the PLC. Due to the cyclic character of the acceleration mechanism the cutter has to be activated at a particular time with high time accuracy. After the cutting action, the stepping motor feeds the ice rod again to prepare the next pellet.

High Speed Boolean Processor FM352-5 with a cycle time $<5 \mu \mathrm{s}$ is selected to manage the synchronisation between stepping motor, cutter and centrifuge.

Five data sets determine the parameters of pellet trains. One data set is composed of: number of pellets, pellet length, repetition rate and number of following data set. The pellet length is determined by the number of steps of the stepping motor. The repetition rate is derived by an integer fraction of the centrifuge rotation frequency.

Three time windows enable the execution of the data sets starting with the dedicated start data set. Using this concept, a wide variation of pellet patterns is provided. The execution of the time windows is interlocked by plasma current and pulse stop provided by the AUG safety system. The High Speed Boolean Processor complies with the requirements.

The temperature control of the cryostat is crucial for stable ice production. The control layout consists of a cryostat, which is cooled down using liquid helium to a bias temperature and an electrical heating stage to get the operation temperature. The control of the electrical power supply is integrated in the PLC using a PI control function. The adjustment of PI controller is challenging due to small heat capacities at the required temperatures.

First results confirmed good ice quality both for $\mathrm{D}_{2}$ and $\mathrm{H}_{2}$, although the system is not designed for the latter.

A sequencer routine is implemented in PLC program to ensure reliable process control of ice production. The time needed to produce ice is reduced by a factor of 2 , which is very important to stay in touch with AUG experimental rhythm.

\section{Pellet arrival prediction and its applications}

The arrival of a pellet into the plasma is to be provided to the Discharge Control System (DCS) for control purposes. Pellet arrival time is deductible from the cutting time and the pellet speed. As a function of repetition rate and pellet speed there is more than only one pellet on the way, thus a simple timer is not appropriate.

A digital delay line suitable to retard the arrival signal for the requested number of subsequent pellets is developed. This signal is transferred to the DCS. The Delay Line is realized by a counter whose cycle time is given by the required delay in conjunction with a FIFO storing the timestamps of the incoming signal. The integration into the PLC system to derive automatically the time of flight from the pellet speed is in progress.

Pellet injection during ECRH heated discharges leads to gyrotron safety shut down due to reflected power at the high density cut-off layer of the ablating pellet is observed. Notching of gyrotron heating using pellet arrival signal generated by centrifuge control eliminates this problem. The arrival of a pellet is announced $3 \mathrm{~ms}$ in advance. The DCS cycle time jitter is $\sim 2 \mathrm{~ms}$, the pellet arrival jitter $\sim 0,5 \mathrm{~ms}$. The typical ablation time of pellet in plasma is $\sim 1 \mathrm{~ms}$. An Off-time of $6 \mathrm{~ms}$ turned out to be sufficient.

Hence the ECRH duty cycle is $94 \%$ at a Pellet repetition rate of $10 \mathrm{~Hz}$ and decreases to $70 \%$ at a rep. rate of $50 \mathrm{~Hz}$. The heating power is reduced by this factor.

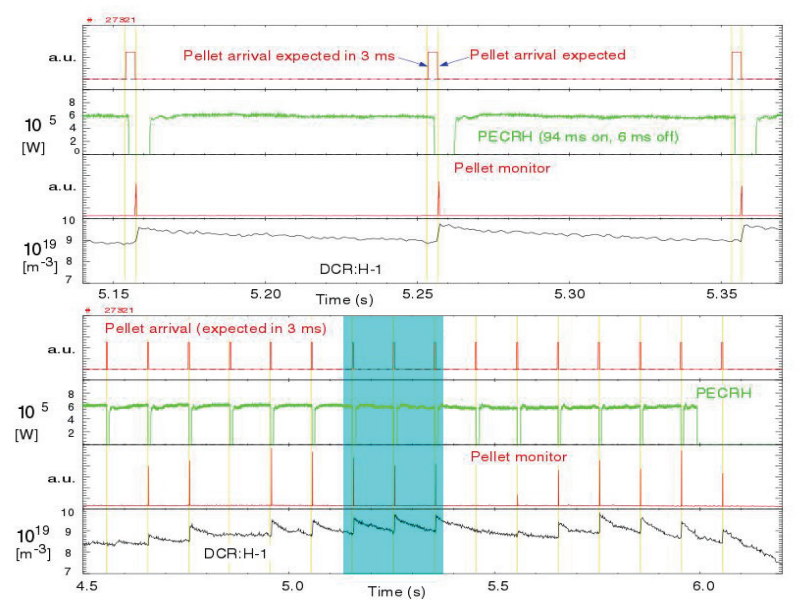

Fig. 3: Notching of ECRH using pellet arrival signal. $6 \mathrm{~ms}$ off-time is sufficient to cover the pellet ablation period.

Furthermore, the availability of pellet arrival signal enables triggering of any system designated to pellet related events, e.g. video diagnostics.

\section{Results of new PLC concept}

Variation of repetition rate of pellet injection means variation of mass flux into the plasma. This can be used for example to assist a density ramp followed by a plateau phase to keep conditions constant. For demonstration 5 data sets are executed consecutively. Figure 4 shows a frequency ramp, with repetition rates of 
$7 \mathrm{~Hz} / 10 \mathrm{~Hz} / 14 \mathrm{~Hz} / 20 \mathrm{~Hz} / 35 \mathrm{~Hz}$. The centrifuge rotation frequency is $140 \mathrm{~Hz}$; the repetition rate is generated by an integer fraction of the rotation frequency. Thus the divider is $20 / 14 / 10 / 7 / 4$. Using this option, smoothly stepped ramps can be realized. A high delivery efficiency into the plasma is achieved $(>90 \%)$.
Variation of pellet mass can be realized using the option to change the number of steps of the stepping motor which feeds the ice into the cutter. Fig. 5 shows a proof of this new functionality. Length variation is reasonably possible within limits; short pellets are prone to break.

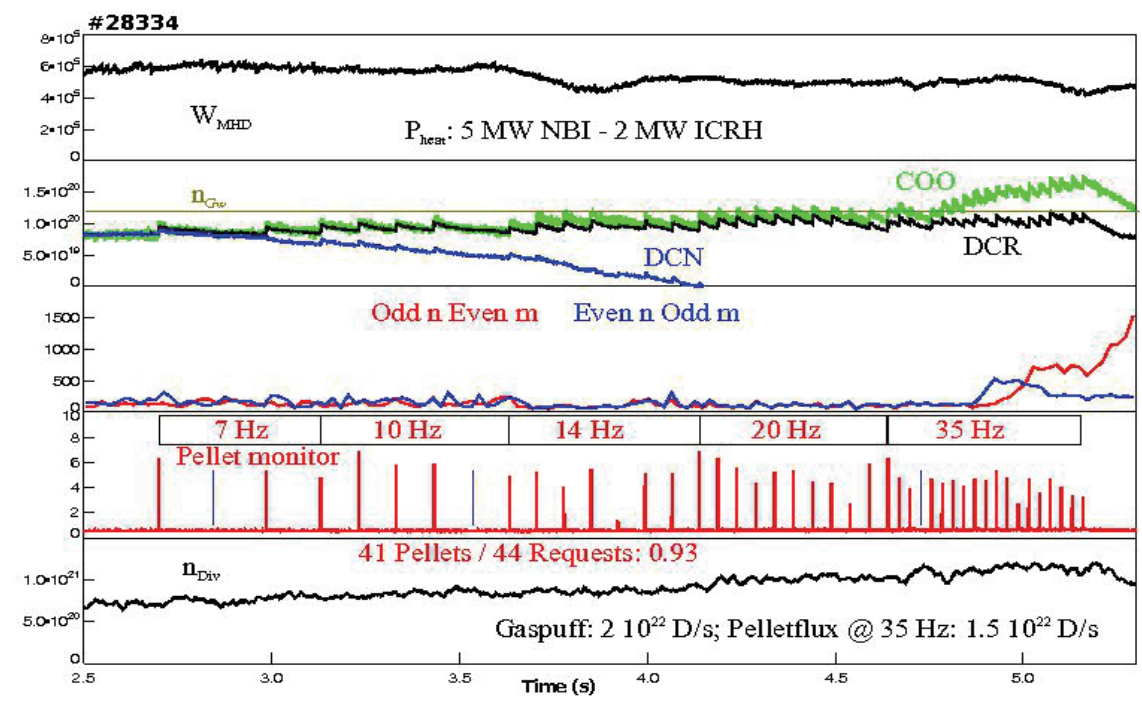

Fig. 4: Frequency ramp with increasing repetition rate (5 steps) using this option, smoothly stepped ramps or particular pattern of repetition rate is possible. In this example, a clear density gain is created (curve $\mathrm{COO}$, measured by $\mathrm{CO}_{2}$ interferometer). The DCN interferometer suffers from fringe loss (DCN and DCR curve).

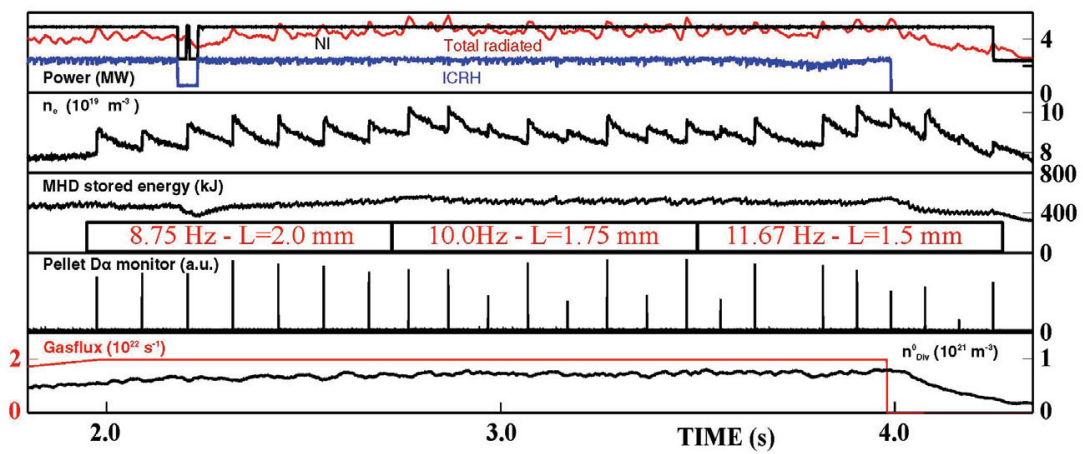

Fig. 5: Variation of pellet mass in AUG\#28393: 3 data sets executed consecutively, increase of the repetition rate is compensated by reduction of pellet length to keep the mass flow constant.

\section{Conceptual study for long pulse operation}

In the actual system, the length of the ice rod is limited due to the technical design of the piston extruder and the storage cryostat. A simple scaling of the existing design concept is not possible. The maximum deuterium inventory in the vacuum chamber to meet explosion protection requirements inhibits this approach. Hence, the number of pellets is limited to $\sim 100$ depending on the length of the pellets. For pacing studies, a long pulse operation is desirable. The centrifuge system is the only acceleration system capable to provide pellet trains with low time jitter needed for ELM pacing operation [2]. Thus this acceleration system has to be kept for long pulse operation too. In order to check the options to introduce a new long pulse capable pellet source in the centrifuge acceleration system, a system study was carried out by the company PELIN LLC.

The new system will require a capability to produce 400 pellets with a repetition rate up to $40 \mathrm{~Hz}$. The pellet size has to be cubical shape with $\mathrm{L}=1.0$ to $1.7 \mathrm{~mm}$. The cycle time (time between blow of not used ice rod and production of a new ice rod) needs to be not more than $10 \mathrm{~min}$. A standby time of at least $30 \mathrm{~min}$ (waiting for the discharge) is required.

The basic idea, elaborated in this study, is to separate the cryostat chamber from the acceleration chamber to get stable vacuum conditions and meet safety requirements for $\mathrm{D}_{2}$ - inventory. In addition, the new pellet source is to be replaceable (by the existing one) on a day to day basis. The ice rod will be continuously produced during a pellet train by a screw extruder with 
an adjustable orifice. To comply with the concept of separated chambers, the ice rod is driven through a duct to the pellet fabrication system. The pellet is cut there out of the moving ice rod. For this purpose, a ferrule which separates the ice rod from the acceleration chamber is moved back and forth just in the moment of cutting the pellet. The unused ice has to be evaporated and exhausted to a recovery system. The ice rod fills in the duct and acts as a vacuum seal.

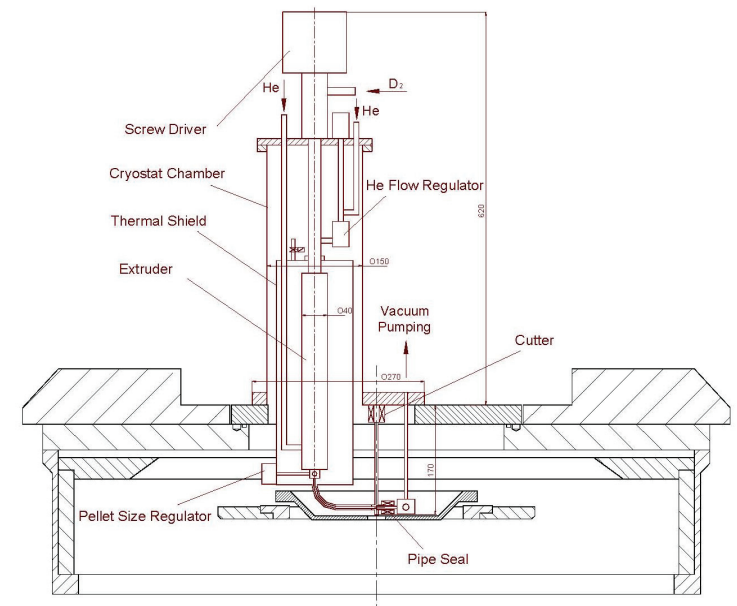

Fig. 6: Conceptual study for long pulse operation, ice production chamber is separated from acceleration chamber. Pellet source adapted to existing centrifuge acceleration system.

\section{First advanced applications in AUG}

\subsection{Advanced fuelling in ELM mitigated discharges}

(Resonant) magnetic perturbations -(R)MP- created by internal coils can result in ELM mitigation once a critical density is achieved [3].

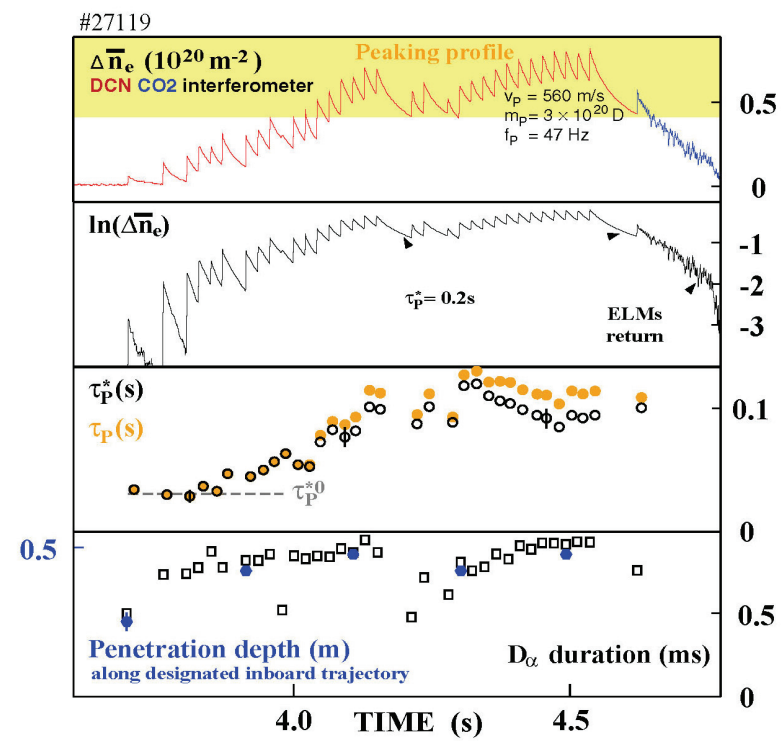

Fig. 7: Fuelling in ELM mitigated discharges; the strong density increase is caused by a pronounced enhancement of the pellet particle sustainment time. This is indicated by the decay time $\tau_{\mathrm{p}}{ }^{*}$ as calculated from the line averaged density evolution. There is also some influence by changing the pellet penetration depth and profile peaking.
The gas puff to reach ELM mitigation can be largely replaced by more efficient pellet fuelling. Successful fuelling replacement is achieved with a total gas puff reduction of about 5 times the applied pellet flux. Experiments to identify the largest density enhancement shows a line averaged density $\mathrm{n}_{\mathrm{e}}=1.8 \times 10^{20} \mathrm{~m}^{-3}$, corresponding to a factor 1.5 of Greenwald density [4]. The largest density enhancement can be achieved by using large pellets and deepest possible penetration and deposition. ELM mitigated phase reduces particle loss, pellet particle confinement time increases. Figure 7 shows results of a dedicated experiment. So far, no physical limits were detected.

\subsection{ELM control}

In an ELM mitigated phase under appropriate (R)MP suppression conditions, strong pellet-induced perturbation triggers no ELM and can be considered as a probe for edge stability. In the absence of such conditions, e.g. in the ramp-up phase after the L-H transition, pellets trigger an ELM. Triggering an ELM immediately after L-H transition might be useful to avoid a long ELM-free phase that is often terminated by a particularly larger type-I ELM.

In non-nuclear phase of ITER, $\mathrm{D}_{2}$ - pellets are not acceptable to mitigate ELM activity, in particular during the Ip ramp-up phase. We demonstrated the option to use $\mathrm{H}_{2}$ - pellets.

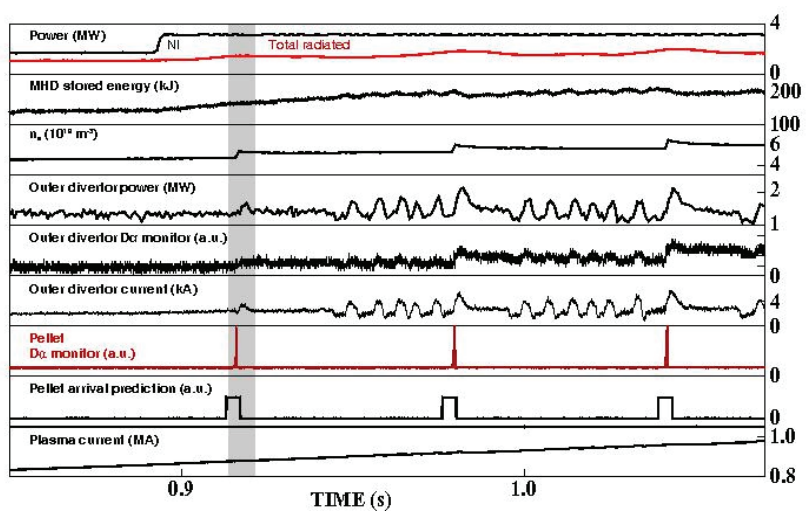

Fig. 8: $\mathrm{H}_{2}$ - pellets in an experiment to trigger the first ELM in AUG He- Plasma AUG \#28481.

\section{Conclusions}

The enhanced high speed inboard pellet fuelling system, based on a centrifuge accelerator, was substantially modernized and successfully put back into operation. Further new technical features were introduced for regular operation and will enable extended and new experimental options, already confirmed by first applications.

\section{References}

[1] A. Lorenz et al., Fusion Engineering and Design 69 (2003) 15-20

[2] B. Plöckl et al., Fusion Engineering and Design 86 (2011) 1022-1025

[3] W. Suttrop et al., Phys. Rev. Lett. 106, 225004 (2011)

[4] P.T. Lang et al., Nucl. Fusion 52 (2012) 023017 